\title{
Heating of the fuel mixture due to viscous stress ahead of accelerating flames in deflagration-to-detonation transition
}

\author{
Damir Valiev ${ }^{1,2}$, Vitaly Bychkov ${ }^{1}$, V'yacheslav Akkerman ${ }^{1}$, Lars-Erik Eriksson ${ }^{3}$ \\ and Mattias Marklund ${ }^{1}$ \\ ${ }^{1}$ Department of Physics, Umeå University, SE-901 8\%, Umeå, Sweden \\ ${ }^{2}$ Department of Materials Science and Engineering, \\ Royal Institute of Technology, SE-100 44 Stockholm, Sweden \\ ${ }^{3}$ Department of Thermo- and Fluid Dynamics, \\ Chalmers University of Technology, SE-412 96 Göteborg, Sweden
}

\begin{abstract}
The role of viscous stress in heating of the fuel mixture in deflagration-to-detonation transition in tubes is studied both analytically and numerically. The analytical theory is developed in the limit of low Mach number; it determines temperature distribution ahead of an accelerating flame with maximum achieved at the walls. The heating effects of viscous stress and the compression wave become comparable at sufficiently high values of the Mach number. In the case of relatively large Mach number, viscous heating is investigated by direct numerical simulations. The simulations were performed on the basis of compressible Navier-Stokes gas-dynamic equations taking into account chemical kinetics. In agreement with the theory, viscous stress makes heating and explosion of the fuel mixture preferential at the walls. The explosion develops in an essentially multi-dimensional way, with fast spontaneous reaction spreading along the walls and pushing inclined shocks. Eventually, the combination of explosive reaction and shocks evolves into detonation.
\end{abstract}




\section{INTRODUCTION}

Deflagration-to-detonation transition (DDT) is one of the most important and least understood problems in combustion science. It is well known that a deflagration wave propagating from a closed tube end may spontaneously accelerate and trigger an explosion in the fresh fuel mixture, which goes over to detonation [1, 2, 3, 4, 45, 6]. The mechanism of DDT was described qualitatively already by Shelkin [2]. According to the Shelkin explanation, thermal expansion in the burning process produces a strong flow in the unburnt fuel mixture, which becomes non-uniform because of non-slip at the tube walls. The non-uniform flow makes the flame curved, which increases the flame surface and hence the volumetric burning rate; it makes the flow even stronger and leads to flame acceleration. An accelerating flame pushes compression waves and shocks, the temperature in the fuel mixture increases, the reaction goes even faster, until explosion happens ahead of the flame front and evolves into detonation.

For a long time it was a general belief that DDT is impossible without artificial turbulence generation. Since turbulence and turbulent burning are not so well-understood yet, it presents a major obstacle in developing a theory of flame acceleration and DDT. Only recently a constructive idea was suggested that DDT may be achieved even in a laminar flow in tubes with adiabatic walls [7, 8]. Though it is difficult to obtain adiabatic walls in a real experiment, these conditions may be easily imitated in direct numerical simulations [7, 8]. Starting with this idea, the analytical theory of laminar flame acceleration was developed in [9, 10]; the theory was quantified by extensive numerical simulations. The idea of laminar flame acceleration allowed also direct numerical simulations of DDT, e.g. see [7, 11, 12] and a recent review [6]. The simulations demonstrated many interesting details of the DDT; at present it is not quite clear, which of them are intrinsic to DDT, making them the backbone of the process, and which are just supplementary effects of minor importance. One of these details is heating of the fuel mixture due to viscous stress, which makes the explosion preferential close to the walls. This is different from the classical Shelkin scenario of DDT with heating produced mainly by the compression/shock waves.

A quantitative analytical theory of explosion triggering by an isentropic compression wave ahead of an accelerating flame was developed recently in [13]. This theory disregarded influence of the viscous stress. However, an important role for viscous stress is in line (at 
least, ideologically) with the model of the DDT, based on hydraulic resistance, see [14] and references therein. This model had another shortcoming; it was one-dimensional and did not take into account Shelkin acceleration, which is an intrinsic part of DDT. Thus we came to the question, how strong the role of viscous stress is in heating of the fuel mixture and in DDT, taking into account the multi-dimensional Shelkin mechanism of this process. The purpose of the present paper is to answer this question. The paper considered only the geometry of smooth tubes, with no obstacles at the walls.

We studied the role of viscous stress both analytically and numerically. The analytical study is based on the theory of laminar flame acceleration [9]. The theory works excellently for low Mach numbers, typical for the beginning of flame acceleration, about $M a=10^{-3}-$ $10^{-2}$. We found temperature profile in the flow ahead of the flame front with maximal temperature increase achieved at the walls. We show that, at low values of the Mach number, the role of viscous heating is extremely small, it scales as $M a^{2}$. By comparison, heating due to the compression wave scales as $M a$. However, temperature at the walls grows faster in time than at the axis. According to the theoretical estimates, the heating effects of viscous stress and of the compression wave become comparable as soon as the Mach number (defined using total burning rate) reaches values above 0.6. Another interesting point is that heating due to viscous stress does not depend on the Reynolds number, at least as long as the Mach number is sufficiently low. When the Mach number becomes about 0.05 and higher, the theory [9] works only qualitatively, providing order-of-magnitude estimates.

In that range of Mach numbers we investigated the role of viscous heating by direct numerical simulations. The simulations were performed on the basis of compressible NavierStokes gas-dynamic equations taking into account chemical kinetics. It was again established that the fuel mixture is heated stronger at the walls than along the tube axis. In agreement with the analytical theory, we found that heating effects of viscous stress and compression wave become comparable at large values of the Mach number; viscous heating makes explosion of the fuel mixture preferential at the walls. The explosion develops in an essentially multi-dimensional way, with fast spontaneous reaction spreading along the walls and pushing rather strong shocks inclined with respect to the tube axis. Eventually, the combination of explosive reaction and shocks evolves into detonation.

The present paper is organized as follows: in Section II we develop the analytical theory of heating due to viscous stress; in Section III we describe the details of the numerical 
simulations; in Section IV we present and discuss the results obtained; the paper is concluded with a short summary.

\section{THEORY OF HEATING DUE TO VISCOUS STRESS AHEAD OF ACCELER- ATING FLAMES}

We consider the same geometry as in [9]: a laminar flame propagates in a two-dimensional (2-D) tube of half-width $R$ with adiabatic walls and non-slip at the walls as shown schematically in Fig. 1) Burning matter expands as it passes the flame front; density ratio of the fuel mixture $\rho_{f}$ and the burnt gas $\rho_{b}$ is typically rather large, $\Theta=\rho_{f} / \rho_{b}=5-8$. Because of the thermal expansion, a flame propagating from the closed tube end pushes the unburnt fuel mixture and generates a flow. It was shown in [9] that the stream ahead of the flame may be closely approximated by a plane-parallel flow along the walls $\mathbf{u}=\hat{\mathbf{e}}_{z} u_{z}(x, t)$.

The normal planar flame velocity $U_{f}$ provides a natural scaling of velocity dimension for the problem. However, the total burning rate $U_{w}$ is different from $U_{f}$ : it shows how much fuel mixture is consumed per unit time by the whole flame front and how much energy is produced. In the standard 2-D model of an infinitely thin flame front, the relative increase in the burning rate is simply equal to the increase in the total length of the flame front. The propagating flame generates a flow, which is not uniform because of non-slip at the walls. Friction retards the gas close to the walls, while flow velocity at the tube axis is larger than the average one. The non-uniform velocity profile distorts the flame shape, which leads to increase in $U_{w}$ and hence to flame acceleration. At sufficiently small values of the Mach number, neglecting the starting transitional period, the flame has been shown to accelerate exponentially [9]

$$
U_{w} / U_{f}=\exp \left(\sigma U_{f} t / R\right)
$$

For 2-D flow, the dimensionless acceleration rate $\sigma$ was found to be [9]

$$
\sigma=\frac{(R e-1)^{2}}{4 R e}\left(\sqrt{1+\frac{4 R e \Theta}{(R e-1)^{2}}}-1\right)^{2}
$$

where the value $R e=U_{f} R / \nu$ plays the role of the Reynolds number for the problem. For large values of the Reynolds number, Eq. (2) predicts decrease of the acceleration rate

$$
\sigma=\Theta^{2} / R e .
$$


In [9], the Navier-Stokes equation was solved as

$$
u_{z} / U_{f}=(\Theta-1) \exp \left(\sigma U_{f} t / R\right) \frac{\cosh \mu-\cosh (\mu x / R)}{\cosh \mu-\mu^{-1} \sinh \mu},
$$

where $\mu=\sqrt{\sigma R e}$. For large values of the Reynolds number we have $\mu=\Theta$, and the flow resembles qualitatively a combination of two boundary layers at the walls separated by the main almost uniform stream, see Fig. 2, Paper [9] demonstrated a very good agreement of the theory with direct numerical simulations of acceleration flames.

Here we find the temperature increase in the fuel mixture ahead of the accelerating flame using the results above. Similar to [9], we adopt the limit of incompressible flow, which holds in the case of a sufficiently small Mach number. In that case the equation of thermal conduction in the fuel mixture is [15]

$$
\frac{\partial T}{\partial t}+\mathbf{u} \cdot \nabla T=\chi \nabla^{2} T+\frac{\nu}{2 C_{P}}\left(\frac{\partial u_{i}}{\partial x_{k}}+\frac{\partial u_{k}}{\partial x_{i}}\right)^{2} .
$$

In the case of a shear flow $\mathbf{u}=\hat{\mathbf{e}}_{z} u_{z}(x, t)$, with $T=T(x, t)$, Eq. (5) reduces to

$$
\frac{\partial T}{\partial t}=\chi \frac{\partial^{2} T}{\partial x^{2}}+\frac{\nu}{C_{P}}\left(\frac{\partial u_{z}}{\partial x}\right)^{2}
$$

We introduce standard scalings $R, U_{f}$ and $R / U_{f}$ as units of length, velocity and time. In that case we work with the dimensionless values $(\eta ; \xi)=(x ; z) / R, \tau=U_{f} t / R, w=u_{z} / U_{f}$, and the dimensionless temperature $\vartheta=T / T_{0}-1$. Taking into account the relation for sound speed in a politropic gas $c_{s 0}^{2}=(\gamma-1) C_{P} T_{0}$ with heat capacity $C_{P}$ and adiabatic exponent $\gamma$, we rewrite Eq. (6) as

$$
\frac{\partial \vartheta}{\partial \tau}=\frac{1}{\operatorname{PrRe}} \frac{\partial^{2} \vartheta}{\partial \eta^{2}}+(\gamma-1) \frac{M a_{0}^{2}}{R e}\left(\frac{\partial w}{\partial \eta}\right)^{2} .
$$

where $M a_{0}=U_{f} / c_{s 0}$ is the Mach number defined with the help of the planar flame velocity. The last term in Eq. (17) is specified by the theory [9] with

$$
\left(\frac{\partial w}{\partial \eta}\right)^{2}=(\Theta-1)^{2} \mu^{2} \exp (2 \sigma \tau) \frac{\sinh ^{2}(\mu \eta)}{\left(\cosh \mu-\mu^{-1} \sinh \mu\right)^{2}} .
$$

We evaluate the possible effect of heating keeping in mind the limit of large Reynolds number with $\sigma=\Theta^{2} / R e, \mu=\Theta$, see Eq. (3). Maximal heating happens close to the wall. Due to symmetry, we may consider only the wall at $\eta=1$, where $\sinh (\mu \eta) \approx \cosh (\mu \eta) \approx \frac{1}{2} \exp (\mu \eta)$. 
Such an approximation holds with exponential accuracy $\exp (-\Theta)<<1$. In that case we have close to the wall (at $1-\eta<<1$ )

$$
\left(\frac{\partial w}{\partial \eta}\right)^{2}=\frac{(\Theta-1)^{2}}{(\mu-1)^{2}} \mu^{4} \exp [2 \sigma \tau+2 \mu(\eta-1)],
$$

and $\partial w / \partial \eta \approx 0$ at the channel axis $\eta=0$. Then Eq. (7) reduces to

$$
\frac{\partial \vartheta}{\partial \tau}=\frac{1}{\operatorname{Pr} \operatorname{Re}} \frac{\partial^{2} \vartheta}{\partial \eta^{2}}+(\gamma-1) \frac{M a_{0}^{2}(\Theta-1)^{2}}{\operatorname{Re}(\mu-1)^{2}} \mu^{4} \exp [2 \sigma \tau+2 \mu(\eta-1)] .
$$

A particular solution to Eq. (10) may be written as $\vartheta_{p} \propto \exp [2 \sigma \tau+2 \mu(\eta-1)]$, which leads to

$$
\vartheta_{p}=-\frac{(\gamma-1) \operatorname{Pr}(\Theta-1)^{2} \mu^{2}}{2(2-\operatorname{Pr})(\mu-1)^{2}} M a_{0}^{2} \exp [2 \sigma \tau+2 \mu(\eta-1)] .
$$

Here we take into account that $\mu=\sqrt{\sigma R e}$. A complete solution to Eq. (10) is a superposition of the particular solution Eq. (11) and the solution to an auxiliary equation

$$
\frac{\partial \vartheta}{\partial \tau}=\frac{1}{\operatorname{Pr} R e} \frac{\partial^{2} \vartheta}{\partial \eta^{2}}
$$

which also grows in time as $\vartheta \propto \exp (2 \sigma \tau)$ so that

$$
2 \sigma \operatorname{Pr} \operatorname{Re\vartheta }=\frac{\partial^{2} \vartheta}{\partial \eta^{2}}
$$

Solution to the auxiliary Eq. (13) may be found as

$$
\vartheta_{a}=\left[A_{1} \exp (k \eta)+A_{2} \exp (-k \eta)\right] \exp (2 \sigma \tau),
$$

with unknown amplitudes $A_{1}, A_{2}$ and

$$
k=\sqrt{2 \sigma \operatorname{Pr} R e}=\sqrt{2 \operatorname{Pr}} \mu .
$$

The complete solution to Eq. (10) is a superposition of $\vartheta_{p}$ and $\vartheta_{a}$, Eq. (11) and Eq. (14), satisfying the adiabatic boundary condition at the wall and the symmetry condition at the channel axis

$$
\frac{\partial \vartheta}{\partial \eta}=0, \quad \eta=0,1
$$

Since $\vartheta_{p}$ is exponentially small at the axis $\eta=0$, we find $A_{1}=A_{2}$. Within the same exponential accuracy, the decaying term $A_{2} \exp (-k \eta)$ is negligible close to the wall. Thus we find at the wall $\eta=1$

$$
k A_{1} \exp k=(\gamma-1) \frac{\operatorname{Pr}}{(2-\operatorname{Pr})} \frac{(\Theta-1)^{2} \mu^{3}}{(\mu-1)^{2}} M a_{0}^{2}
$$


which determines the complete solution close to the wall as

$$
\vartheta=\frac{(\gamma-1) \operatorname{Pr}(\Theta-1)^{2} \mu^{2}}{2(2-\operatorname{Pr})(\mu-1)^{2}} M a_{0}^{2} \exp (2 \sigma \tau)\left[\frac{2 \mu}{k} \exp [k(\eta-1)]-\exp [2 \mu(\eta-1)]\right] .
$$

The temperature increase at the walls $\eta=1$ is

$$
\vartheta=\frac{(\gamma-1)}{2} \frac{(\Theta-1)^{2} \mu^{2}}{(\mu-1)^{2}} \frac{\sqrt{P r}}{\sqrt{2}+\sqrt{P r}} M a_{0}^{2} \exp (2 \sigma \tau)
$$

Finally, as an evaluation, we consider the limit of large Reynolds number $\sigma=\Theta^{2} / R e, \mu=\Theta$, which leads to the temperature increase

$$
\vartheta=\frac{(\gamma-1)}{2} \Theta^{2} \frac{\sqrt{P r}}{\sqrt{2}+\sqrt{P r}} M a_{0}^{2} \exp (2 \sigma \tau)=\frac{(\gamma-1)}{2} \Theta^{2} \frac{\sqrt{P r}}{\sqrt{2}+\sqrt{P r}} U_{w}^{2} / c_{s 0}^{2} .
$$

It is interesting that viscous heating at the wall, Eq. (20), does not depend on the Reynolds number. Combination $M a_{w}=M a_{0} \exp (\sigma \tau)=U_{w} / c_{s 0}$ determines the running value of the Mach number for an accelerating flame, which is different from the initial value $M a_{0}=$ $U_{f} / c_{s 0}$. As an example, we take $\Theta=8, \operatorname{Pr}=0.7, \gamma=1.4$, and find the temperature increase due to viscous stress $\vartheta \approx 4.8 M a_{w}^{2}=4.8 U_{w}^{2} / c_{s 0}^{2}$.

We compare this result with the heating due to the compression wave found in [13] as

$$
1+\vartheta=\left(1+\frac{\gamma-1}{2}(\Theta-1) M a_{w}\right)^{2}
$$

Note that the designation $\vartheta$ has a different meaning in the present calculations and in [13]. In the case of small Mach numbers we find

$$
\vartheta=(\gamma-1)(\Theta-1) M a_{w}=(\gamma-1)(\Theta-1) \frac{U_{w}}{c_{s 0}}
$$

Using the same numbers as before we obtain a temperature increase in a compression wave $\vartheta=2.8 U_{w} / c_{s 0}$. Thus, for $\Theta=8, \operatorname{Pr}=0.7, \gamma=1.4$, we should expect a comparable temperature increase due to the compression wave and due to viscous stress at the Mach number about $M a_{w}=U_{w} / c_{s 0} \approx 0.6$, see Eqs. (20), (22). Besides, heating due to the compression wave is mostly a 1D process equally strong at the walls and at the channel axis. As both effects work together, we find preferential heating at the walls. However, we have to remember that the theory of flame acceleration [9] works quantitatively well only for a noticeably subsonic flow, $M a=10^{-3}-10^{-2}$. At such small Mach numbers heating due to viscous stress is negligible, since it scales as $\vartheta \propto M a_{w}^{2}$, while heating in a compression wave 
has another scaling $M a \propto M a_{w}$. At Mach numbers above 0.05 the theory [9] provides only order-of-magnitude estimates. Direct numerical simulations of the present paper show that, at Mach numbers above 0.05, flame acceleration goes much slower than in the exponential regime Eq. (1). The critical value $M a_{w}=U_{w} / c_{s 0} \approx 0.6$ obtained above is also an order-ofmagnitude estimate; it is beyond the rigorous limits of the developed theory. Still, the result Eq. (20) may be useful even at relatively high values of the Mach number. For comparison, temperature increase due to viscous stress in the stationary boundary layer produced by a uniform flow $U_{a}$ is [16]

$$
\frac{\Delta T}{T_{a}}=\frac{\gamma-1}{2} \sqrt{\operatorname{Pr}} \frac{U_{a}^{2}}{c_{s a}^{2}}
$$

Here $T_{a}$ and $c_{s a}$ are the temperature and the sound speed in the uniform flow $U_{a}$ far away from the wall. In the present problem flow velocity in the main stream (e.g. at the axis) plays qualitatively the same role as $U_{a}$. Rewriting Eq. (20) in the same form, we find

$$
\frac{\Delta T}{T_{a}}=\frac{\gamma-1}{2} \frac{\sqrt{P r}}{\sqrt{2}+\sqrt{P r}} \frac{U_{a}^{2}}{c_{s a}^{2}} .
$$

Here we have taken into account that velocity at the axis ahead of an accelerating flame is related to the total burning rate as $U_{a}=\Theta U_{w}$, see Eq. (44). The difference between Eqs. (23) and (24) is then simply in the numerical factor $\sqrt{2}+\sqrt{\operatorname{Pr}}$, which is about 2.25 for $\operatorname{Pr}=0.7$. An exponentially accelerating flame produces about half the temperature increase at the wall compared to that from the stationary boundary layer. Thus one would expect that the temperature increase at $M a_{w}=0.05$ and above is somewhere between predictions of Eqs. (23) and (24). In order to investigate the role of viscous heating at relatively large values of the Mach number we performed direct numerical simulations. We stress once more that both formulas (23) , (24) predict viscous heating independent of the Reynolds number. This

conclusion is of great help in numerical simulations, since it allows investigating the process even for rather low values of the tube width.

\section{BASIC EQUATIONS OF THE DIRECT NUMERICAL SIMULATIONS}

We performed direct numerical simulations of the 2-D hydrodynamic and combustion equations including transport processes (thermal conduction, diffusion, viscosity) and chemical kinetics with an Arrhenius reaction. The equations read

$$
\frac{\partial \rho}{\partial t}+\frac{\partial}{\partial x_{i}}\left(\rho u_{i}\right)=0
$$




$$
\begin{gathered}
\frac{\partial}{\partial t}\left(\rho u_{i}\right)+\frac{\partial}{\partial x_{j}}\left(\rho u_{i} u_{j}+\delta_{i j} p-\gamma_{i j}\right)=0 \\
\frac{\partial}{\partial t}\left(\rho \varepsilon+\frac{1}{2} \rho u_{i} u_{i}\right)+\frac{\partial}{\partial x_{j}}\left(\rho u_{j} h+\frac{1}{2} \rho u_{i} u_{i} u_{j}+q_{j}-u_{i} \gamma_{i j}\right)=0 \\
\frac{\partial}{\partial t}(\rho Y)+\frac{\partial}{\partial x_{i}}\left(\rho u_{i} Y-\frac{\mu}{S c} \frac{\partial Y}{\partial x_{i}}\right)=-\frac{\rho Y}{\tau_{R}} \exp \left(-E / R_{p} T\right)
\end{gathered}
$$

where $Y$ is the mass fraction of the fuel mixture, $\varepsilon=Q Y+C_{V} T$ is the internal energy, $h=Q Y+C_{p} T$ is the enthalpy, $Q$ is the energy release in the reaction, $C_{V}, C_{p}$ are the heat capacities at constant volume and pressure. It was assumed that the heat capacities do not depend on the chemical reaction. The stress tensor $\gamma_{i j}$ and the energy diffusion vector $q_{j}$ take the form

$$
\begin{gathered}
\gamma_{i j}=\mu\left(\frac{\partial u_{i}}{\partial x_{j}}+\frac{\partial u_{j}}{\partial x_{i}}-\frac{2}{3} \frac{\partial u_{k}}{\partial x_{k}} \delta_{i j}\right), \\
q_{j}=-\mu\left(\frac{C_{p}}{\operatorname{Pr}} \frac{\partial T}{\partial x_{j}}+\frac{Q}{S c} \frac{\partial Y}{\partial x_{j}}\right),
\end{gathered}
$$

where $\mu \equiv \rho \nu$ is the dynamic viscosity, $\operatorname{Pr}$ and $S c$ are the Prandtl and Schmidt numbers, respectively. To avoid the Zeldovich (thermal-diffusion) instability we take the unit Lewis number $L e \equiv \operatorname{Pr} / S c=1$, with $\operatorname{Pr}=S c=0.75$. The dynamical viscosity is $\mu=1.7 \times$ $10^{-5} \mathrm{Ns} / \mathrm{m}^{2}$. The fuel-air mixture and burnt matter are assumed to be a perfect gas with a constant molar mass $m=2.9 \times 10^{-2} \mathrm{~kg} / \mathrm{mol}$, with $C_{V}=5 R_{p} / 2 \mathrm{~m}, C_{p}=7 R_{p} / 2 \mathrm{~m}$, where $R_{p} \approx 8.31 \mathrm{~J} /(\mathrm{mol} \cdot \mathrm{K})$ is the perfect gas constant. The adiabatic index is $\gamma \equiv C_{P} / C_{V}=1.4$. The equation of state is

$$
P=\rho R_{p} T / m
$$

We used the initial temperature of the fuel mixture $T_{f}=300 \mathrm{~K}$, initial pressure $P_{f}=10^{5} \mathrm{~Pa}$, and initial expansion ratio $\Theta_{0}=8$. The initial Mach number is $M a_{0}=U_{f} / c_{s 0}=0.04$. Eq. (28) describes a single irreversible reaction of the first order, where the reaction rate obeys the Arrhenius law with the activation energy $E_{a}$ and the factor of time dimension $\tau_{R}$. In the simulations, the value of $U_{f}$ is determined by the choice of thermal-chemical parameters such as $E_{a}, \tau_{R}$, and the energy release in the reaction $Q=\left(\Theta_{0}-1\right) C_{P} T_{f}$. In order to have better resolution of the reaction zone, we took $E_{a} / R_{p} T_{f}=32$ in the simulations. Note that the 
process of viscous heating addressed in our paper does not depend on the activation energy. The factor $\tau_{R}$ was adjusted to obtain a particular value of the planar flame velocity $U_{f}$ by solving the associated eigen-value problem. The flame thickness is conventionally defined as

$$
L_{f} \equiv \frac{\mu}{\operatorname{Pr} \rho_{f} U_{f}}
$$

where $\rho_{f}=1.16 \mathrm{~kg} / \mathrm{m}^{3}$ is the initial mixture density. However, we would like to stress that the value (32) is just a thermal-chemical parameter of length dimension in the problem; the characteristic size of the burning zone may be an order of magnitude larger [17].

In the studies of flame acceleration [9, 10], the main parameter of simulations was the tube width $2 R$, which controlled the Reynolds number. In the present paper we are interested in thermal heating due to viscous stress. According to the present theory and the classical results [16], viscous heating does not depend on the Reynolds number (or this dependence is extremely weak). For this reason, to study the effect, it is sufficient to perform simulations only for one channel width. The channel width was set to $2 R=40 L_{f}$, which implied the Reynolds number related to the planar flame velocity $R e=U_{f} R / \nu=R / L_{f} \operatorname{Pr}=26.7$. The Reynolds number related to the flow $R e=\left\langle u_{z}\right\rangle 2 R / \nu$ may be larger by several orders of magnitude due to flame acceleration and thermal expansion of the burning gas. We took non-slip and adiabatic boundary conditions at the channel walls:

$$
\mathbf{u}=0, \quad \hat{\mathbf{n}} \cdot \nabla T=0 .
$$

where $\hat{\mathbf{n}}$ is a normal vector at the wall. At the open channel end non-reflecting boundary conditions are used; the boundary conditions were tested in [17]. We used two types of initial conditions: 1) a hemispherical flame "ignited" at the channel axis at the closed end of the channel, or 2) a planar flame front.

The internal structure of the flame imitated the analytical solution by Zel'dovich and Frank-Kamenetskii [3]. Particularly, for the hemispherical case it was given by

$$
\begin{gathered}
T=T_{f}+T_{f}(\Theta-1) \exp \left(-\sqrt{x^{2}+z^{2}} / L_{f}\right), \quad \text { if } \quad z^{2}+x^{2}>r_{f}^{2} . \\
T=\Theta T_{f}, \quad \text { if } \quad z^{2}+x^{2}<r_{f}^{2} \\
Y=\frac{\Theta-T / T_{f}}{\Theta-1}, \quad P=P_{f}, \quad \mathbf{u}=0 .
\end{gathered}
$$


Here $r_{f}$ is the radius of initial flame ball at the closed end of the channel. For an initially planar flame shape Eq. (34) is replaced by

$$
T=T_{f}+T_{f}(\Theta-1) \exp \left[-\left(z-z_{0}\right) / L_{f}\right],
$$

if $z>z_{0}$, with Eq. (35) for $z<z_{0}$ ( $z_{0}$ denotes the initial flame position).

We simulated flame dynamics and DDT using a 2-D Cartesian Navier-Stokes solver with chemical reactions developed at Volvo Aero. The code is based on the cell-centered finitevolume scheme developed by L.-E. Eriksson [18, 19]. The numerical method has proved to be robust for modelling different kinds of complex reacting flows. The TVD-limiting is used where applicable to prevent overshoots in flow field properties in regions of high gradients. The code has been validated by solving various hydrodynamic problems [18, 19, 20], and was utilized successfully in simulations of laminar flames at different conditions of burning $[9,10,12,17,21,22,23,24,25,26]$.

The grids used in present simulations were non-uniform with higher resolution around the flame front and pressure waves and lower resolution in other parts of the calculation domain. Grid resolution varied gradually from one zone to another along the tube. Using this method we avoided processing a too large number of cells. Square cells of the size $0.2 L_{f}$ were used in the flame domain to ensure isotropic propagation of the curved flame in $\mathrm{x}$ and y directions. In [17] we demonstrated that such a grid is sufficient to resolve even a strongly curved flame front. As an illustration, in Fig. 3(a) we present the profiles of the main variables inside the flame front: the temperature distribution $\left(T-T_{f}\right) /\left(T_{b}-T_{f}\right)$, the mass fraction $Y$ and the reaction rate $A=\left(\rho Y / \tau_{R}\right) \exp \left(-E_{a} / R_{p} T\right)$ scaled by its maximal value. We observe that the thermal-chemical parameter $L_{f}$ defined by Eq. (32) is much smaller than the real flame thickness. According to Fig. 3(a), the characteristic length scale of the temperature profile is about $(4-5) L_{f}$, while the width of the active reaction zone is about $L_{f}$. Taking the grid size of $0.2 L_{f}$ in $z$-direction we obtained about five grid points inside the active reaction zone and 20-25 grid points inside the flame.

A sketch of the calculation grid is shown in Fig. 3(b), The calculation grid is modified periodically in the course of calculation, so that the sizes and positions of all domains in Fig. 3(b) change dynamically, following the flame front and pressure waves. Thus the leading compression wave can never reach the open tube end during a calculation. Cell size was $0.4 L_{f}$ around the front of the leading pressure wave. Splines of the third order are used 
for re-interpolation of the flow variables during periodic grid reconstruction to preserve the second order accuracy of the numerical scheme. We performed several test simulation runs with different resolution between 0.1 and $0.2 L_{f}$ in the flame domain. The tests demonstrated that the difference in the flame velocity, pre-detonation time and distance does not exceed $10 \%$ for different resolutions.

\section{SIMULATION RESULTS}

We performed direct numerical simulation of flame dynamics and detonation triggering in a tube of width $2 R=40 L_{f}$ with non-slip walls. The flame propagated from the closed tube end to the open one; a semi-infinite tube was imitated by using non-reflecting boundary conditions at the open end and by adjusting the mesh to the leading pressure wave. We started the simulations either with a hemispherical flame ignited at the tube axis or with a planar flame front. Fig. 4 4shows velocity of the combustion front in the laboratory reference frame of the tube, $U_{\text {lab }} / U_{f}$, in the case of hemispherical ignition; the velocity was calculated as the average over the reaction front. Flame dynamics involves several velocity parameters, which have quite different physical meaning and may differ quantitatively by one or two orders of magnitude. First, we have a planar flame velocity $U_{f}$, which characterizes local normal velocity of the flame front relative to the moving fuel mixture (at least in the model of an infinitely thin front). The value $U_{f}$ works as a standard velocity scaling in the problems of flame dynamics. To be accurate, $U_{f}$ depends on thermal and chemical parameters of the fuel mixture, which vary in the process of detonation triggering. Here $U_{f}$ designates the initial planar flame velocity. Second, we have the burning rate $U_{w}$, which specifies total amount of fuel mixture burnt per unit time (see also Section II). The burning rate $U_{w}$ may exceed $U_{f}$ by several orders of magnitude in the process of flame acceleration. Still, the value $U_{w}$ does not describe flame propagation in any particular reference frame except for the case of stationary or statistically-stationary curved flames like in [22, 23, 26, 27, 28].

Flame propagating from the closed end pushes a strong flow in the fuel mixture. In the incompressible case, average velocity of the flow is $\left\langle u_{z}\right\rangle=(\Theta-1) U_{w}$, where averaging is performed over the channel cross-section. The flow drifts the flame, and the drift velocity is different for different parts of the flame front. For this reason, the burning rate $U_{w}$ is

also different from flame velocity in the laboratory reference frame, with respect to the tube 
walls, $U_{l a b}$. We stress that Fig. 4 presents flame velocity in the laboratory reference frame, $U_{l a b}$, not the burning rate $U_{w}$. In the case of an essentially subsonic flame, we have a simple relation $U_{l a b}=\Theta U_{w}$, which may also provide an estimate in the case of finite Mach numbers. Different velocity parameters imply different Mach numbers as well, which we designate by $M a_{0}=U_{f} / c_{s 0}, M a_{w}=U_{w} / c_{s a}, M a_{l a b}=U_{l a b} / c_{s a}$; label "a" refers to the position at the channel axis just ahead of the flame front. Taking a rather low initial Mach number related to the planar flame velocity $M a_{0}=0.04$ with $\Theta=8$, we have immediately $M a_{l a b}=0.32$, which is not so low. Soon after ignition, in a short time about $(0.2-0.3) R / U_{f}$, burning rate increases by order of magnitude in the process of precursor acceleration [29, 30]. As a result, quite quickly the Mach number $M a_{l a b}$ becomes comparable to unity. We also point out that $M a_{w}, M a_{l a b}$ take into account variations of the sound speed in the fuel mixture in time and space; scaled sound speed $c_{s a} / U_{f}$ is shown by the dashed line in Fig. 4(a),

Transition from one combustion regime to another (flame-explosion-detonation) may be clearly seen by drastic changes in the front velocity. In the flame regime, we can see front acceleration, which involves two different physical mechanisms. First, we observe a short period of precursor acceleration, related to the hemispherical geometry of flame ignition. This type of acceleration is possible even in the case of friction-free walls. Precursor acceleration goes noticeably faster than the Shelkin mechanism, but this process is limited in time. Precursor acceleration for a cylindrical tube was investigated experimentally in [29] and numerically in [30]. The paper [30] presented also the analytical theory of the process. In the present geometry of a 2-D channel the precursor acceleration is somewhat different from the cylindrical case; still, the basic features of the process remain the same.

The precursor acceleration is followed by acceleration due to the Shelkin mechanism produced by thermal expansion and non-slip at the walls. The theory of the Shelkin acceleration in incompressible flows has been developed in [9, 10]. However, in the present case Mach number $M a_{l a b}$ is comparable to unity or above, and the incompressible theory of flame acceleration describes the process only qualitatively. A dashed line in Fig. 4(a) shows the exponential growth predicted by the incompressible theory Eq. (1); it goes well above the numerical results as soon as the precursor acceleration is over. Investigation of the compressibility effects on flame acceleration is beyond the scope of the present paper; this is work for the future. Still, as a preliminary result, Fig. 4(b) shows the flame velocity in log-log variables. In order to elucidate the transition effects related to a particular 
geometry of flame ignition, we have performed two simulation runs: that of a flame ignited at the tube axis and one for a flame ignited as a planar front. In the first case we have precursor acceleration of the flame front, while in the second case this effect is missing. As we can see, both simulation runs tend to the same asymptotic regime of flame acceleration with $U_{l a b} \propto\left(U_{f} t / R\right)^{n}$, where $n \approx 0.36$. More detailed investigation of the process will be presented elsewhere. It is well known that flame is subsonic; still, this rule applies to the burning rate $U_{w}$, not to $U_{l a b}$. In Fig. 4(b), $U_{l a b}$ crosses the sonic line at the time instant about $U_{f} t / R=30$. This time instant is often called run-up time to supersonic flames [31].

An accelerating flame produces a flow in the fuel mixture, which is non-uniform because of the non-slip boundary conditions. A characteristic velocity profile is shown in Fig. 5: the results of numerical simulations are compared to the predictions of the incompressible theory [9]. The velocity profile is calculated at different time instants just ahead of the flame front and scaled by the maximum velocity value at the tube axis. Both the theory and the present simulations demonstrate the same qualitative structure of the flow, which consists of an almost uniform stream close to the axis and transitional (boundary) layers close to the walls. The velocity profile is in a good agreement with the incompressible case, not only qualitative, but even quantitative. A non-uniform viscous flow implies stress, which is stronger close to the channel walls. As a consequence, one should expect additional heating of the fuel mixture due to the viscous stress, as discussed in Section II. In this Section, our main goal is to investigate the process of viscous heating numerically and to compare it to the theory of Section II.

The temperature increase in the fuel mixture just ahead of the flame front is shown in Fig. 6] as a function of time and at two positions: at the tube axis and at the channel wall. We can see that, within a short time after ignition $U_{f} t / R=0.2-0.3$, the temperature increase is already different from zero, being about $\Delta T / T_{0} \approx 0.25$. This happens because of the leading shock wave, pushed by the flame in the process of precursor acceleration. The flow velocity behind the shock is about $\Delta u_{z} / U_{f} \approx \Theta-1$, and thermal expansion is typically quite strong. Therefore, in spite of a small initial Mach number $M a_{0}=0.04$, the flow velocity is not so small in comparison with sound speed even for a planar flame front, $\Delta u_{z} / c_{s 0}=0.28$. Precursor acceleration makes this value even larger. At the beginning, temperature increase at the walls and at the tube axis is practically the same, which illustrates almost $1 \mathrm{D}$ structure of the compression wave. As time goes on, the flame accelerates, the Mach number of the 
flow increases and heating due to viscous stress becomes noticeable in agreement with the theory of Section II. By the time of explosion, $U_{f} t / R \approx 4.38$, difference between temperature at the axis and at the walls is noticeable; still, this difference is not as strong as predicted by the theory. In order to compare the numerical simulations and the theory quantitatively, in Fig. [7 we plot temperature increase versus Mach number $M a_{l a b}$. In that case we separate heating in the compression wave (at the axis), and the effect of viscous stress as temperature difference between the wall and the axis, $T_{\text {wall }}-T_{\text {axis }}$. Heating in the compression wave at the axis is shown by squares. Temperature at the axis increases almost linearly with the Mach number, in agreement with the theory, Eq. (21), presented by the solid line. The theoretical prediction holds for a 1D isentropic flow; small deviations of the theory and simulations indicate that the flow is not completely 1D and isentropic. Two other solid lines show theoretical predictions for temperature increase due to viscous stress ahead of an exponentially accelerating flame, Eq. (24), and in a stationary boundary layer, Eq. (23). These two formulas present two limiting cases of strong acceleration and no acceleration at all. As we pointed out, at relatively high values of the Mach number, the flame acceleration is much slower than exponential. By this reason it is natural to expect that the numerical points should be somewhere in between the predictions of Eqs. (23) and (24). Indeed, Fig. 7 shows that this is the case; the numerical results come rather close to that of Eq. (24), being a little above the theoretical curve. Only at the end of the simulation run, temperature at the wall increases drastically, which indicates the beginning of the explosion. It is also interesting to look at the temperature distribution in the channel. Fig. 8 compares temperature profiles at different distances ahead of the flame front at the time instant $U_{f} t / R=3.43$ to the theoretical result Eq. (18). The theoretical predictions look quite similar to the numerical results: all the time we observe the effect of viscous heating well-localized at the walls. Still, the theory predicts a self-similar temperature profile independent of position, since it was developed for an exponentially accelerating flame in an incompressible flow. In the numerical simulations flame acceleration is not exponential, the flow is not incompressible, and the temperature profile changes in space and time. As we can see in Fig. 8, the transitional zone of viscous heating is wider close to the flame front, and it becomes more localized far away from the flame.

As we can see, heating due to viscous stress is not large in comparison with that in a compression wave, and, in principle, explosion theoretically could be possible even without 
viscous heating. Fig. 9 presents temperature field snapshots in the area of the accelerating flame from beginning of the explosion till detonation. Prior to the explosion, we can see light blue strips along the walls indicating regions of viscous heating. Still, Arrhenius reactions are extremely sensitive to temperature; even this slight non-uniformity influences strongly the structure of the explosion. The explosion may be observed already at the second snapshot: it develops in the form of two narrow tongues spreading fast along the tube walls from the flame front to the fuel mixture. Temperature in the explosion zone is noticeably higher than it was behind the flame front. The tongues grow in length and width, until the original

flame front becomes engulfed in the explosion. However, even at that time the explosion front remains essentially multi-dimensional: a large amount of the unburned matter separates two explosion tongues. The explosion pushes inclined pressure waves, well seen in Fig. 9 by the light blue color. Intersection of the pressure waves increases temperature at the channel axis, reduces the reaction time, until a bridge is formed between two tongues of the explosion. As two tongues meet, they soon develop into detonation, which can be seen in the last two snapshots of Fig. 9. Meeting of two explosion tongues may be also seen in Fig. 4, as an extremely sharp peak in the velocity dependence. Strictly speaking, this peak is infinitely sharp and high, since it corresponds to a singularity of pocket formation. In Fig. 4, we cut down the peak to make the plot more illustrative. In the present calculations, DDT distance is $330 R$, but this value is, of course, sensitive to a particular chemical kinetics.

\section{SUMMARY}

The purpose of the present paper was to investigate the role of viscous heating in DDT. We solved this problem both analytically and by direct numerical simulations. The analytical theory was developed within the approximation of incompressible flow, which holds at sufficiently low values of the Mach number. On the contrary, the numerical simulations were performed for relatively large Mach numbers. Both theory and numerical simulations demonstrated that the role of viscous heating increases with the Mach number. Viscous stress makes temperature higher at the channel walls in comparison with the axis. Temperature profile in the channel is given by Eq. (18), temperature increase at the wall due to viscous stress is determined by Eq. (24). The theoretical results agree rather well with the numerical simulations, though, when making a comparison, one has to take into account 
much slower flame acceleration at larger values of the Mach number. Even just before the explosion, additional temperature increase due to viscous heating is noticeably smaller than the temperature increase in the compression wave. Thus, viscous heating should be treated only as a supplementary process in DDT. In principle, DDT could be possible with heating provided by the compression wave only in line with the Shelkin scenario [2, 5, 13]. Still, neglecting viscous heating in a theoretical model like [13] may lead to considerable disagreement in the time and distance of detonation triggering predicted by the theory and obtained in experiments/numerical simulations. Viscous heating makes explosion triggering easier and faster because of extreme sensitivity of Arrhenius reactions to temperature variations. In a certain sense, it plays the same role as hot spots: it is not necessary, but helpful. Nonuniform temperature distribution leads to an essentially multi-dimensional structure of the explosion, which pushes inclined pressure waves and eventually develops into detonation.

\section{ACKNOWLEDGEMENTS}

This work was supported by the Swedish Research Council (VR). Numerical simulations were performed at High Performance Computer Center North (HPC2N), Umeå, Sweden, within SNAC project 007-07-25. The authors also wish to thank Arkady Petchenko and Michael Modestov for useful discussions. 
[1] F. A. Williams, Combustion Theory, Benjamin, Redwood City, CA, 1985.

[2] K. I. Shelkin, Zh. Exp. Teor. Fiz. 10, 823 (1940).

[3] Ya. B. Zeldovich, G. I. Barenblatt, V. B. Librovich, G. M. Makhviladze, Mathematical Theory of Combustion and Explosion, Consultants Bureau, New York, 1985.

[4] J. E. Shepherd, J. H. S. Lee, in Major Research Topics in Combustion, p. 439, Springer Verlag, Hampton, VA, 1992.

[5] G. Roy, S. Frolov, A. Borisov, D. Netzer, Prog. En. Combust. Sci. 30, 545 (2004).

[6] E. Oran, V. Gamezo, Combust. Flame 1484 (2007).

[7] L. Kagan, G. Sivashinsky, Combust. Flame 134, 389 (2003).

[8] J. D. Ott, E. S. Oran, J. D. Anderson, AIAA Journal 41, 1391 (2003).

[9] V. Bychkov, A. Petchenko, V. Akkerman, L.-E. Eriksson, Phys. Rev. E 72, 046307 (2005).

[10] V. Akkerman, V. Bychkov, A. Petchenko, L.-E. Eriksson, Combust. Flame 145, 206 (2006).

[11] L. Kagan, D. Valiev, M. Liberman, G. Sivashinsky, in Proceedings of Pulsed and Continuous Detonations, eds. G. Roy, S. Frolov, J. Sinibaldy, Torus Press Ltd, p. 119 (2006).

[12] M. Liberman, G. Sivashinsky, D. Valiev, L.-E. Eriksson, J. Trans. Phenomena 8, 253 (2006).

[13] V. Bychkov, V. Akkerman, Phys. Rev. E 73, 066305 (2006).

[14] I. Brailovsky, G. Sivashinsky, Combust. Flame 122, 492 (2000).

[15] L. D. Landau, E. M. Lifshitz, Fluid Mechanics (Pergamon Press, Oxford, 1989).

[16] H. Schlichting, Theory of a Boundary Layer, Verlag G. Braun, Karlsruhe, 1969.

[17] V. Akkerman, V. Bychkov, A. Petchenko, L.-E. Eriksson, Combust. Flame 145, 675 (2006).

[18] L.-E. Eriksson, A third-order accurate upwind-based finite volume scheme for unsteady compressible viscous flows, VAC Report 9370-154, Volvo Aero Corporation (1993).

[19] L.-E. Eriksson, Development and validation of highly modular solver versions G2DFlow and G3DFlow series for compressible viscous reactive flow, VAC Report 9970-1162, Volvo Aero Corporation (1995).

[20] L.-E. Eriksson, Comp. Meth. Mech. Eng. 64, 95 (1987).

[21] V. Bychkov, M. Liberman, Phys. Rep. 325, 115 (2000).

[22] V. Bychkov, S. Golberg, M. Liberman, L.-E. Eriksson, Phys. Rev. E 54, 3713 (1996).

[23] O. Travnikov, V. Bychkov, M. Liberman, Phys. Rev. E 61, 468 (2000). 
[24] A. Petchenko, V. Bychkov, V. Akkerman, L.-E. Eriksson, Phys. Rev. Lett. 97, 164501 (2006).

[25] A. Petchenko, V. Bychkov, V. Akkerman, L.-E. Eriksson, Combust. Flame 149, 418 (2007).

[26] M. Liberman, M. Ivanov, O. Peil, D. Valiev, and L.-E. Eriksson, Combust. Theory Model. 7, $653(2003)$.

[27] S. Kadowaki, T. Hasegawa, Prog. Energy Combust. Sci. 31, 193 (2005).

[28] A. Petchenko, V. Bychkov, L.-E. Eriksson, and A. Oparin, Combust. Theory Model. 10, 581 (2006).

[29] C. Clanet, G. Searby, Combust. Flame 105, 225 (1996).

[30] V. Bychkov, V. Akkerman, G. Fru, A. Petchenko, L. E. Eriksson, Combust. Flame 150, 263 (2007).

[31] M. Kuznetsov, V. Alekseev, I. Matsukov, S. Dorofeev, Shock Waves 14, 205 (2005). 


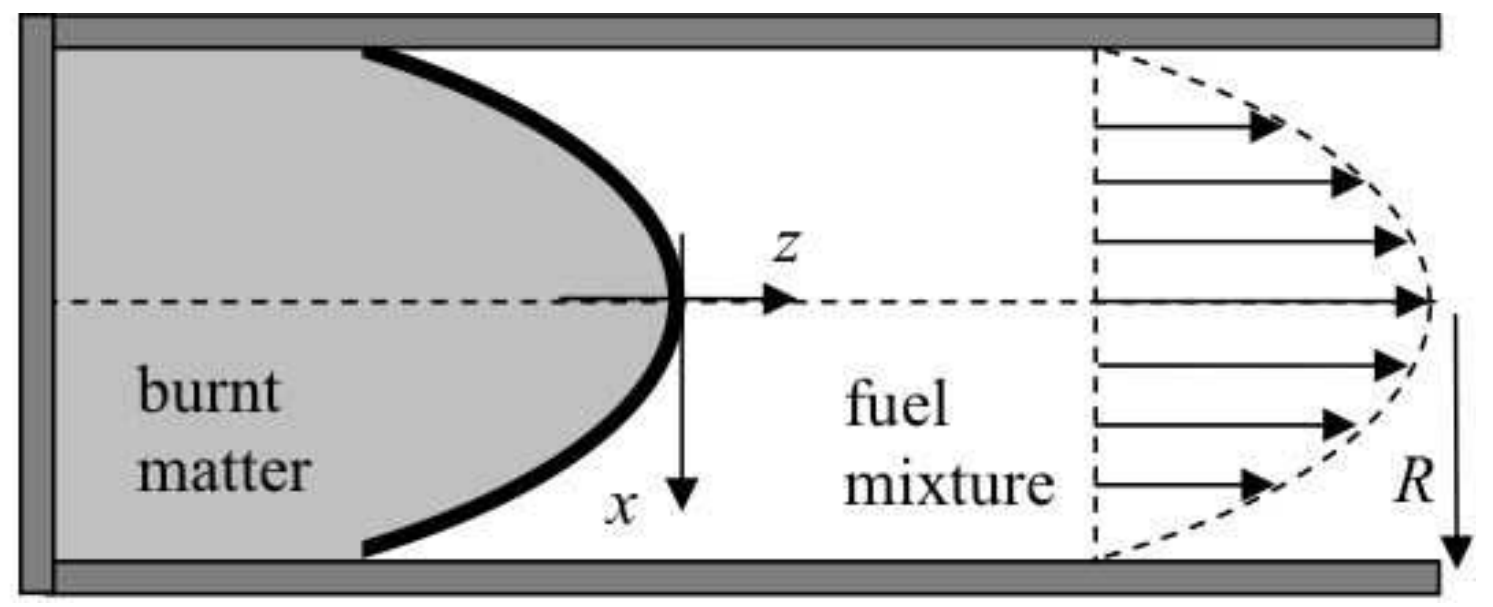

FIG. 1: Flame propagating in a tube with non-slip walls.

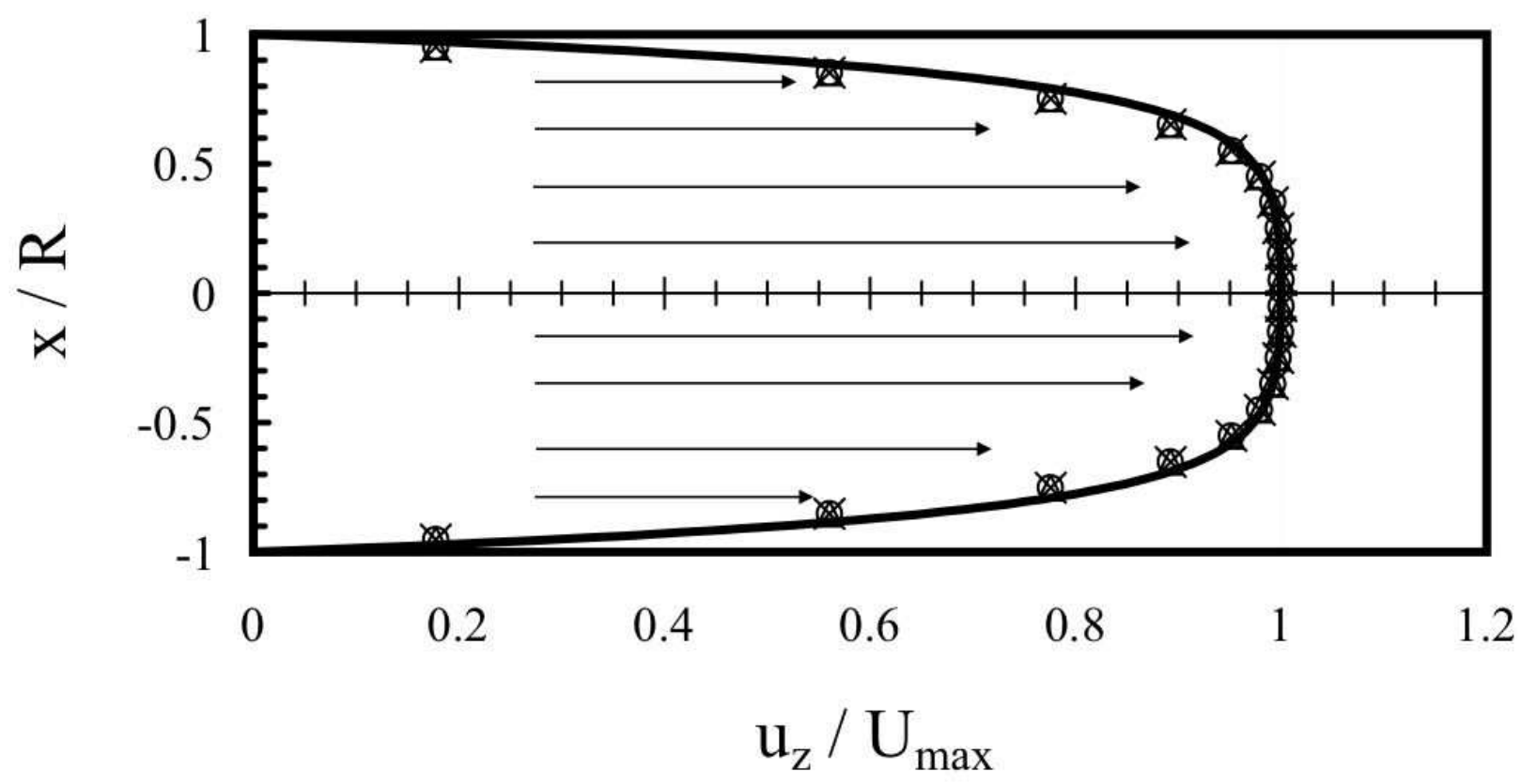

FIG. 2: The velocity profile $u_{z}$ scaled by the amplitude $U_{\max }$. The solid line shows the theoretical result Eq. (4). The markers correspond to the simulation results of [9] for $\operatorname{Re}=50, \operatorname{Pr}=0.5$ at the distances $10 R ; 20 R ; 35 R$ (circles, triangles and crosses) from the flame at the time instance $U_{f} t / R=1.15$. The arrows illustrate the direction of the flow. 


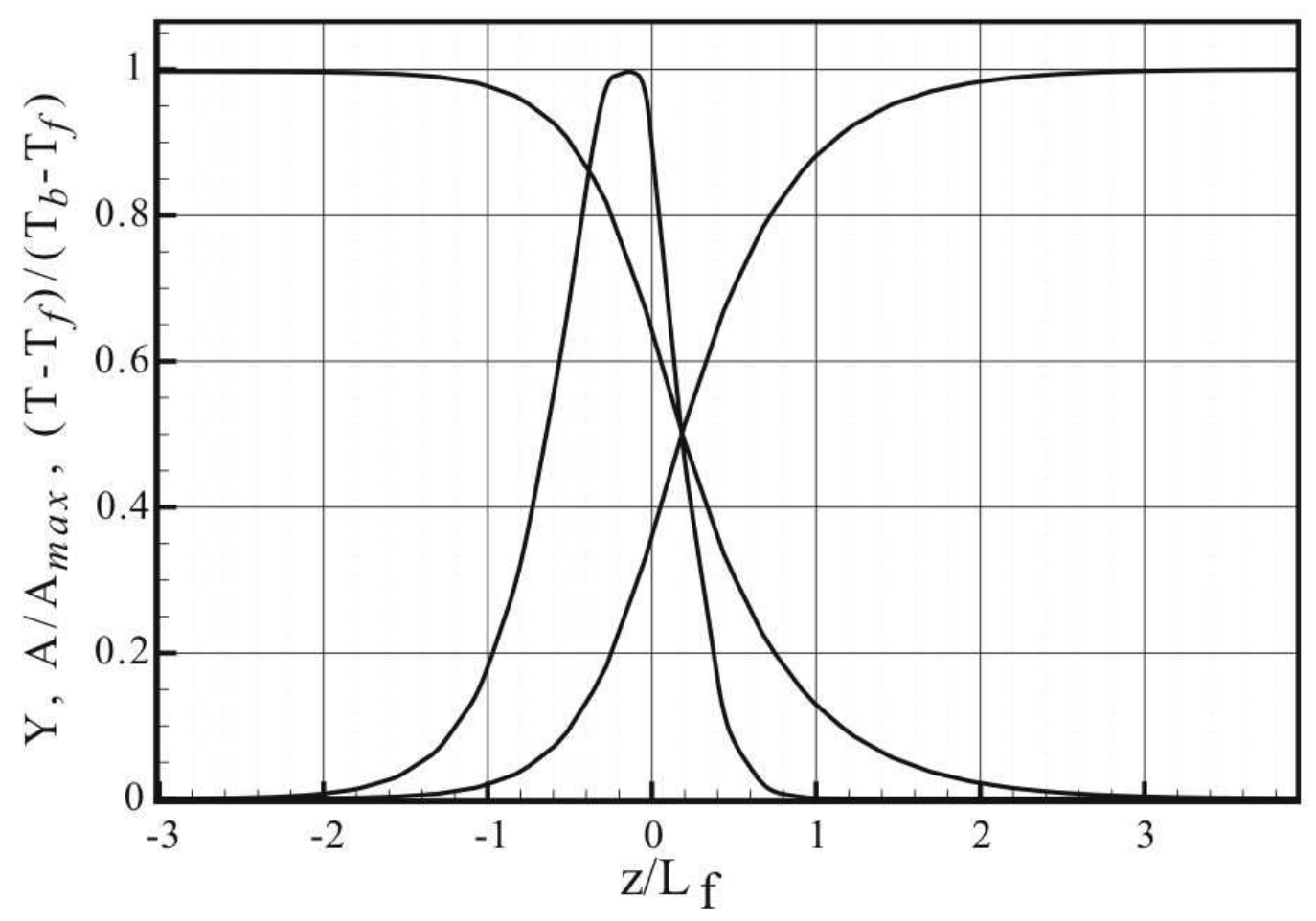

(a)

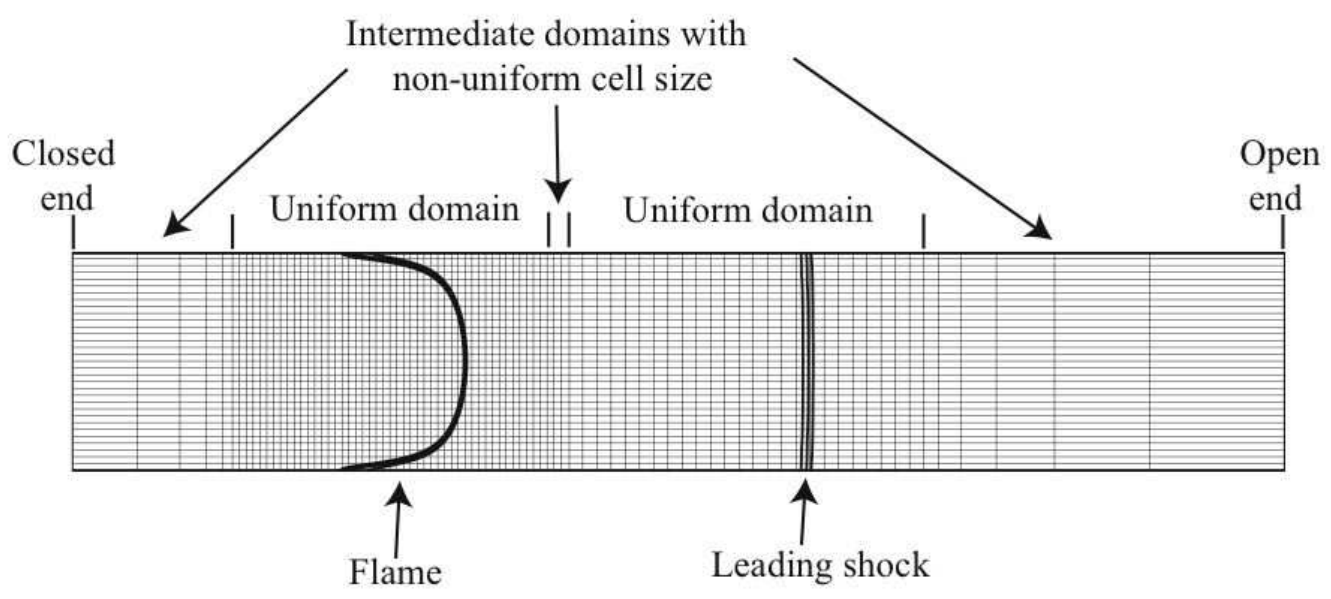

(b)

FIG. 3: Illustration of the grid. (a) Profiles of the scaled temperature $\left(T-T_{f}\right) /\left(T_{b}-T_{f}\right)$, the mass fraction $Y$ and the reaction rate $A=\left(\rho Y / \tau_{R}\right) \exp \left(-E_{a} / R_{p} T\right)$ scaled by its maximal value within the flame front. (b) The adaptive grid used in numerical simulations. 


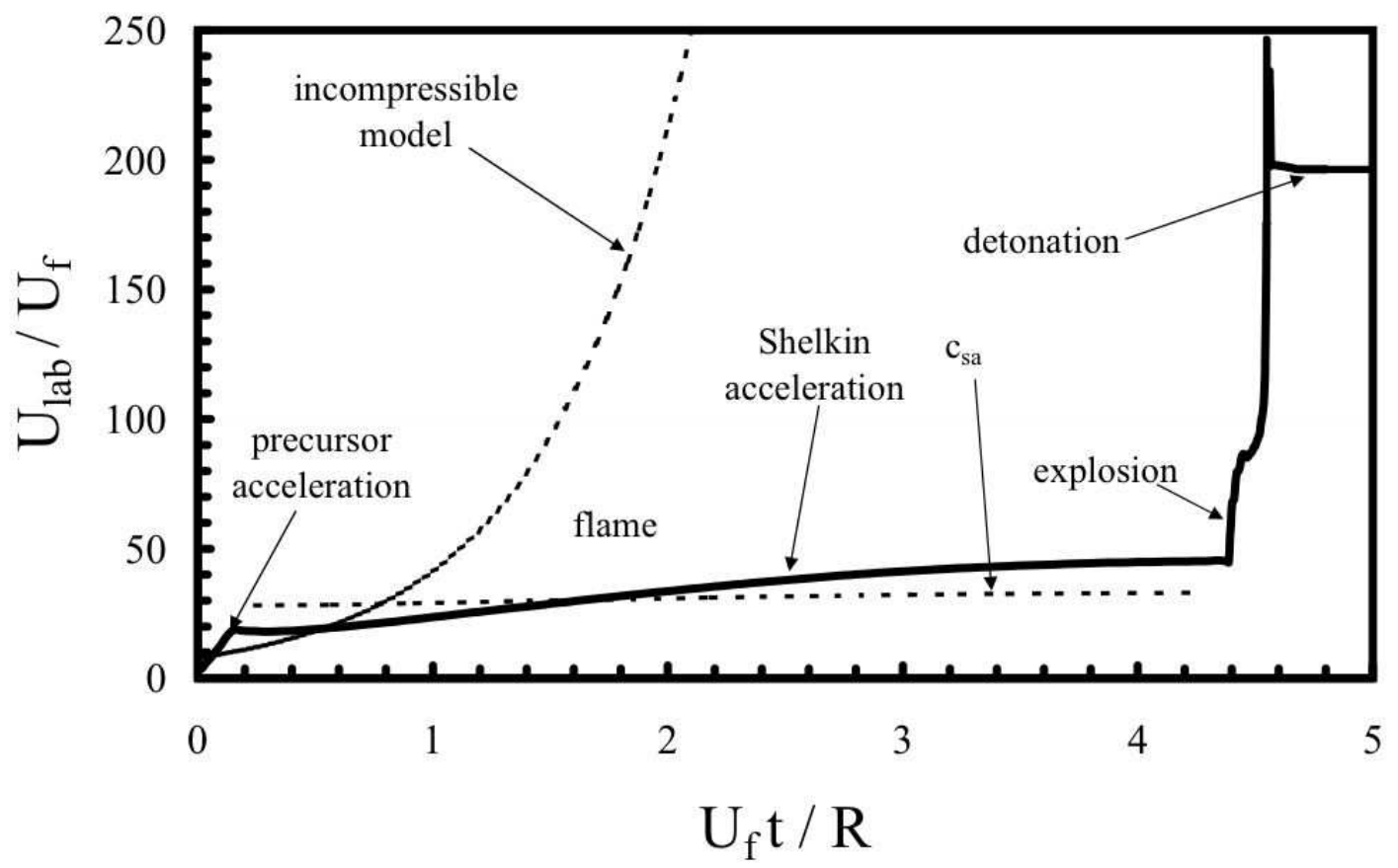

(a)

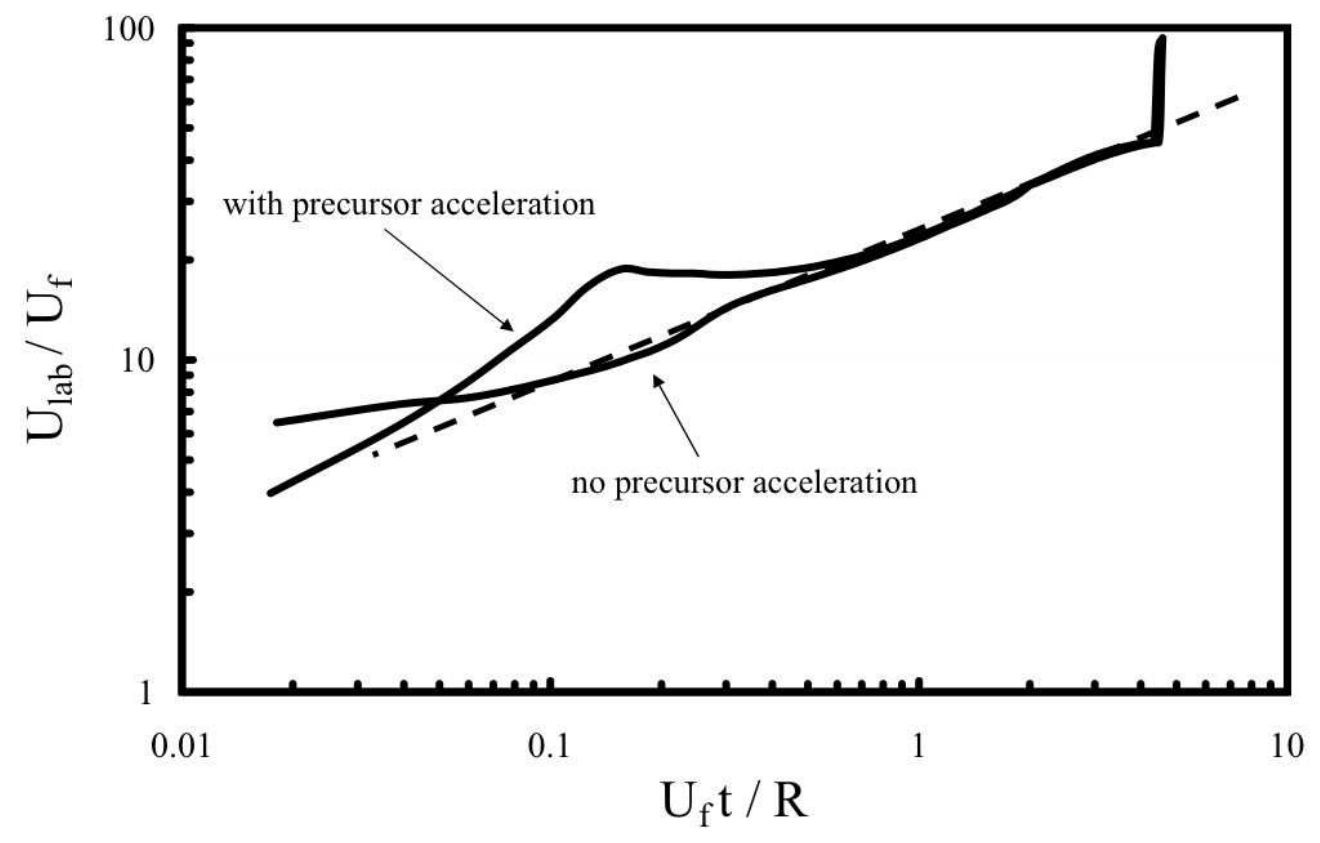

(b)

FIG. 4: (a) Scaled velocity of the reaction front (flame-explosion-detonation) in the laboratory reference frame, $U_{l a b} / U_{f}$. One dashed line presents theoretical predictions for the incompressible flow. The other dashed line shows scaled sound speed, $c_{s a} / U_{f}$. (b) The same plot in $\log -\log$ variables for a flame ignited at the channel axis (with precursor acceleration) and as a planar front (no precursor acceleration). 


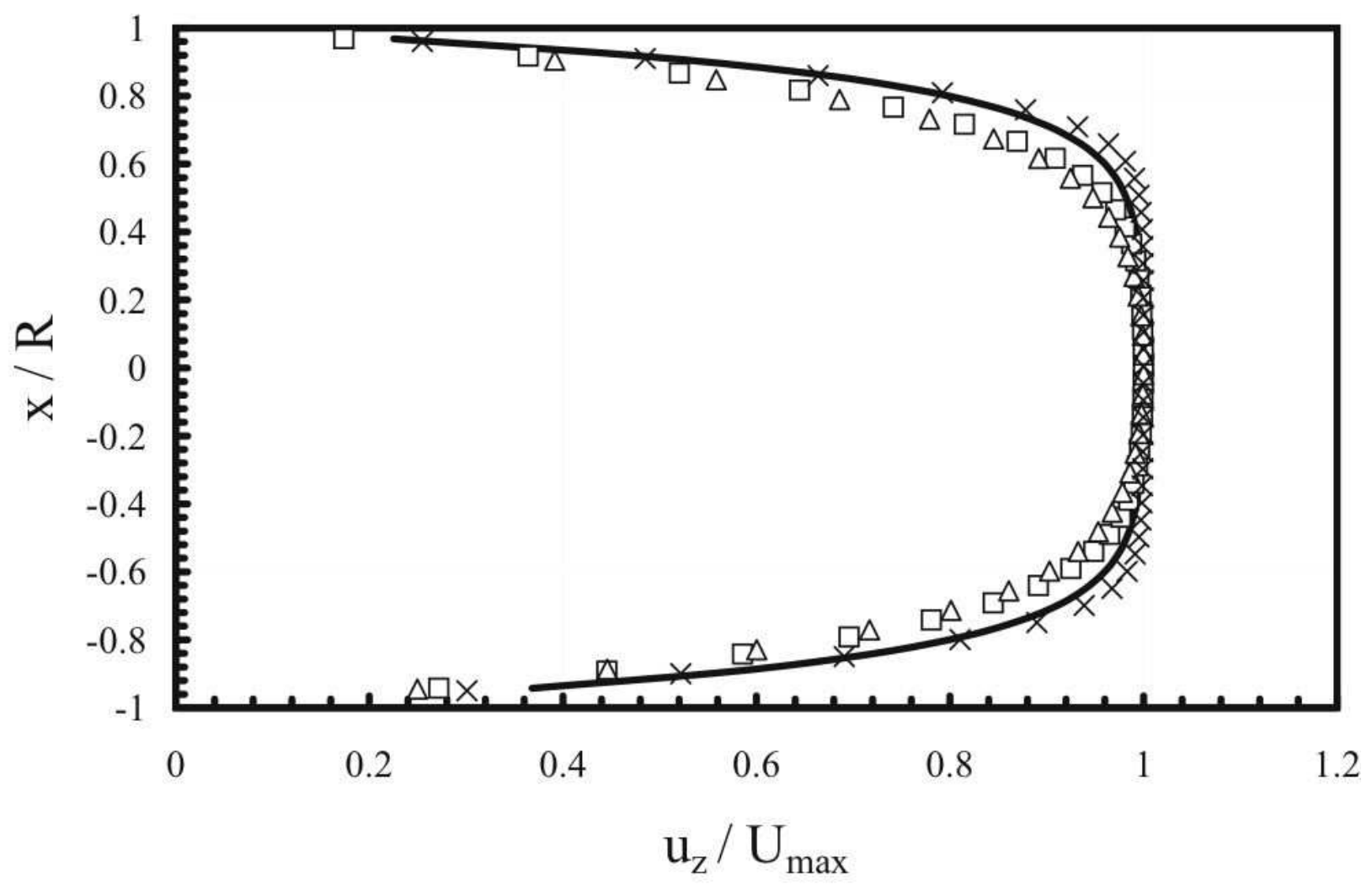

FIG. 5: The velocity profile $u_{z}$ scaled by the amplitude $U_{\max }$. The solid line shows the theoretical result Eq. (4). The markers correspond to the present simulation results just ahead of the flame front at the time instants $U_{f} t / R=0.92 ; 1.74 ; 3.43$. 


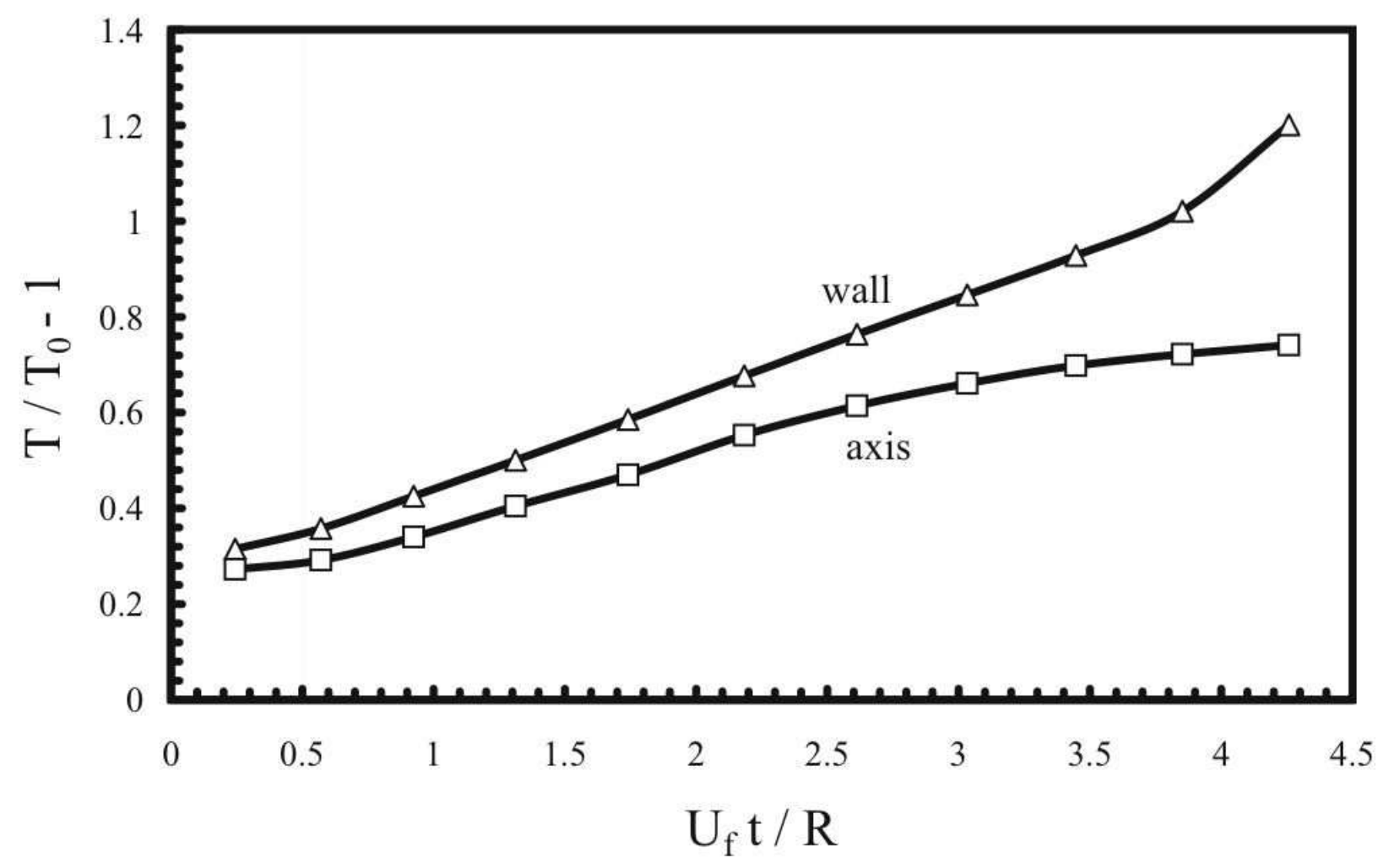

FIG. 6: Temperature increase at the wall and at the channel axis versus time. 


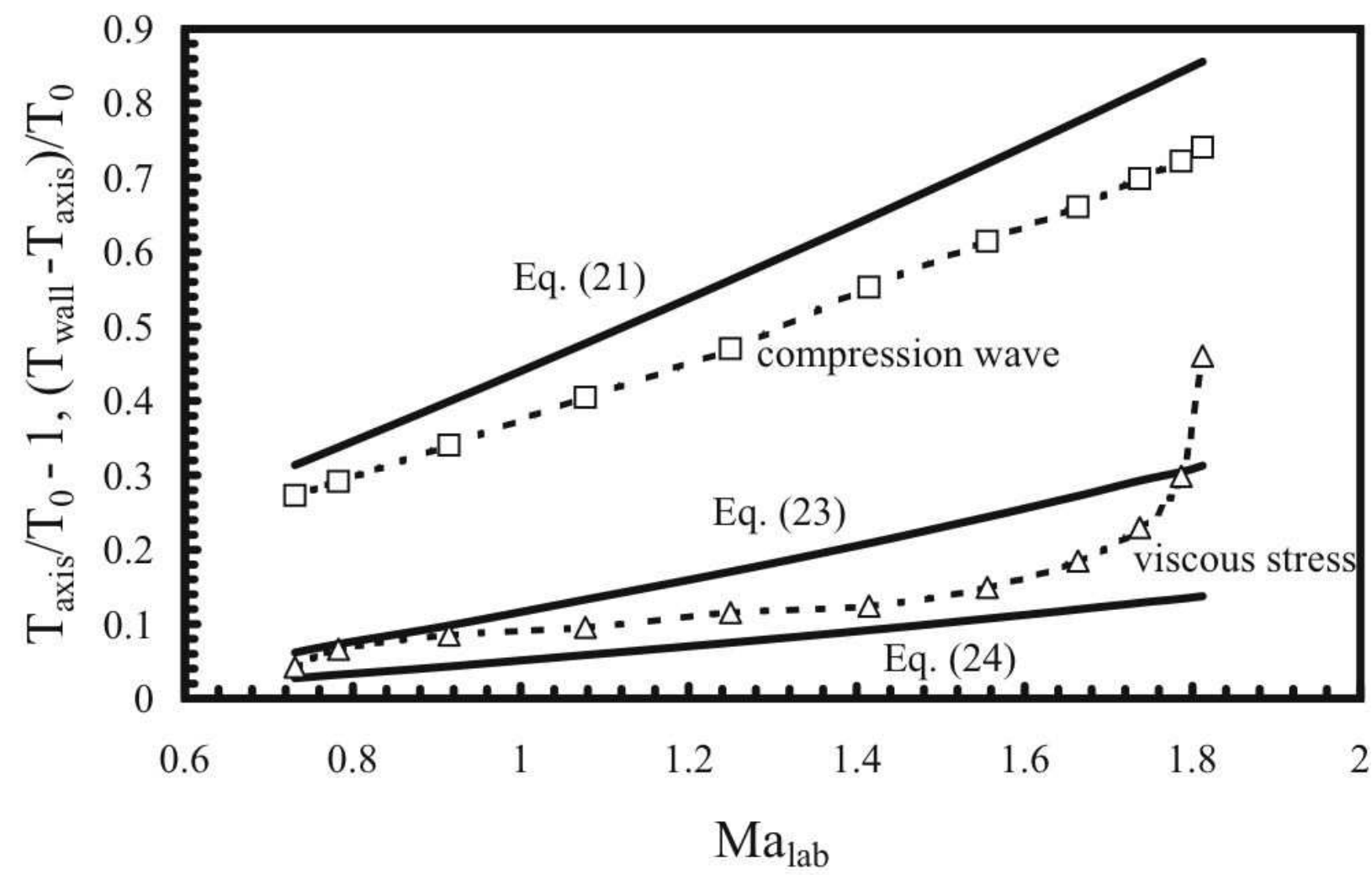

FIG. 7: Temperature increase versus Mach number in the laboratory reference frame. Solid lines show theoretical predictions for temperature increase in the compression wave at the axis, Eq. (21), and additional increase due to viscous stress at the wall, Eqs. (23), (24). The dashed lines and the markers show respective numerical results. 


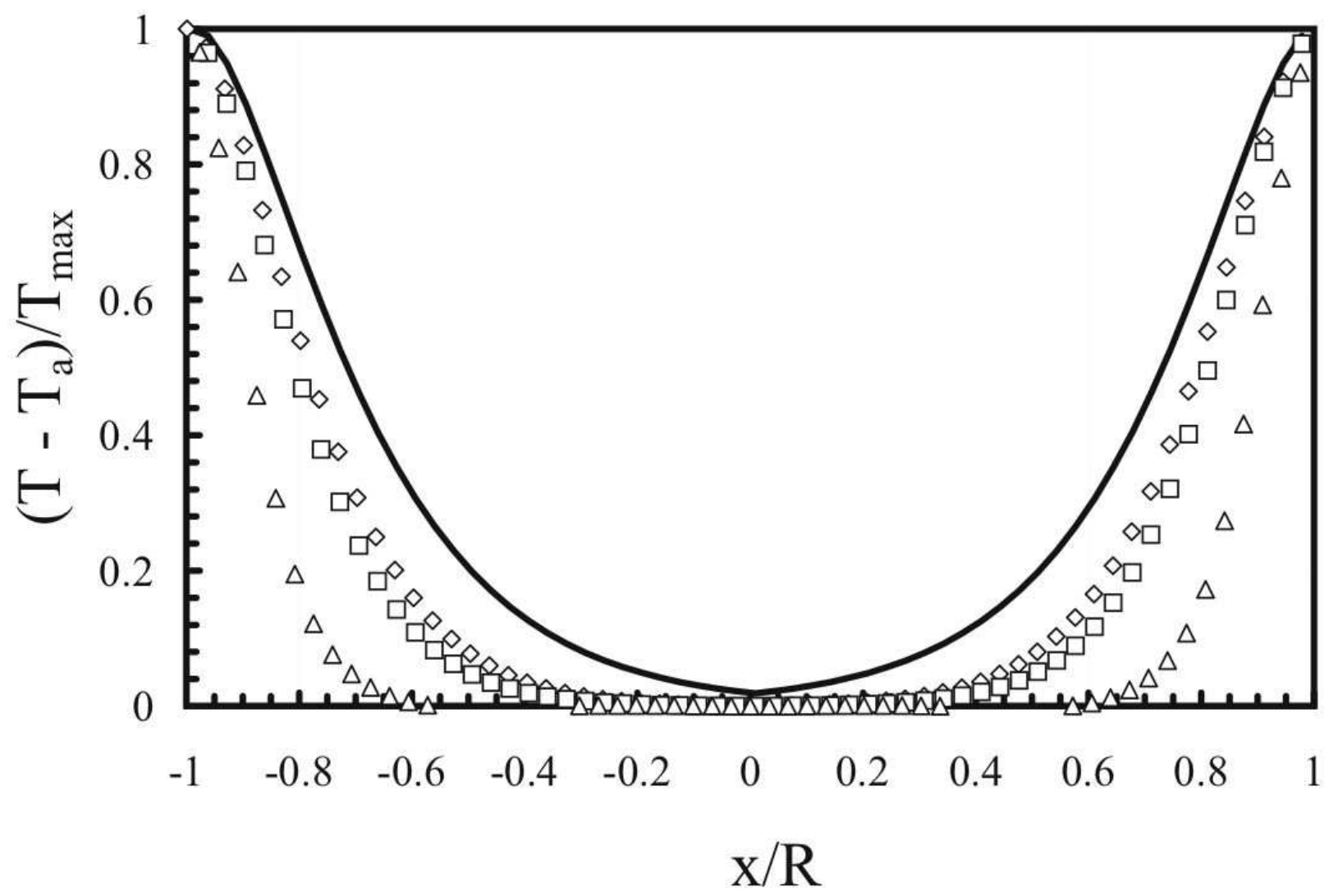

FIG. 8: Scaled temperature profile $\left(T-T_{\text {axis }}\right) /\left(T_{\text {wall }}-T_{\text {axis }}\right)$. The solid line shows the theoretical result Eq. (18). The markers correspond to the present simulation results at the distances $(6 ; 425 ; 930) L_{f}$ ahead of the flame front at the time instant $U_{f} t / R=3.43$. 


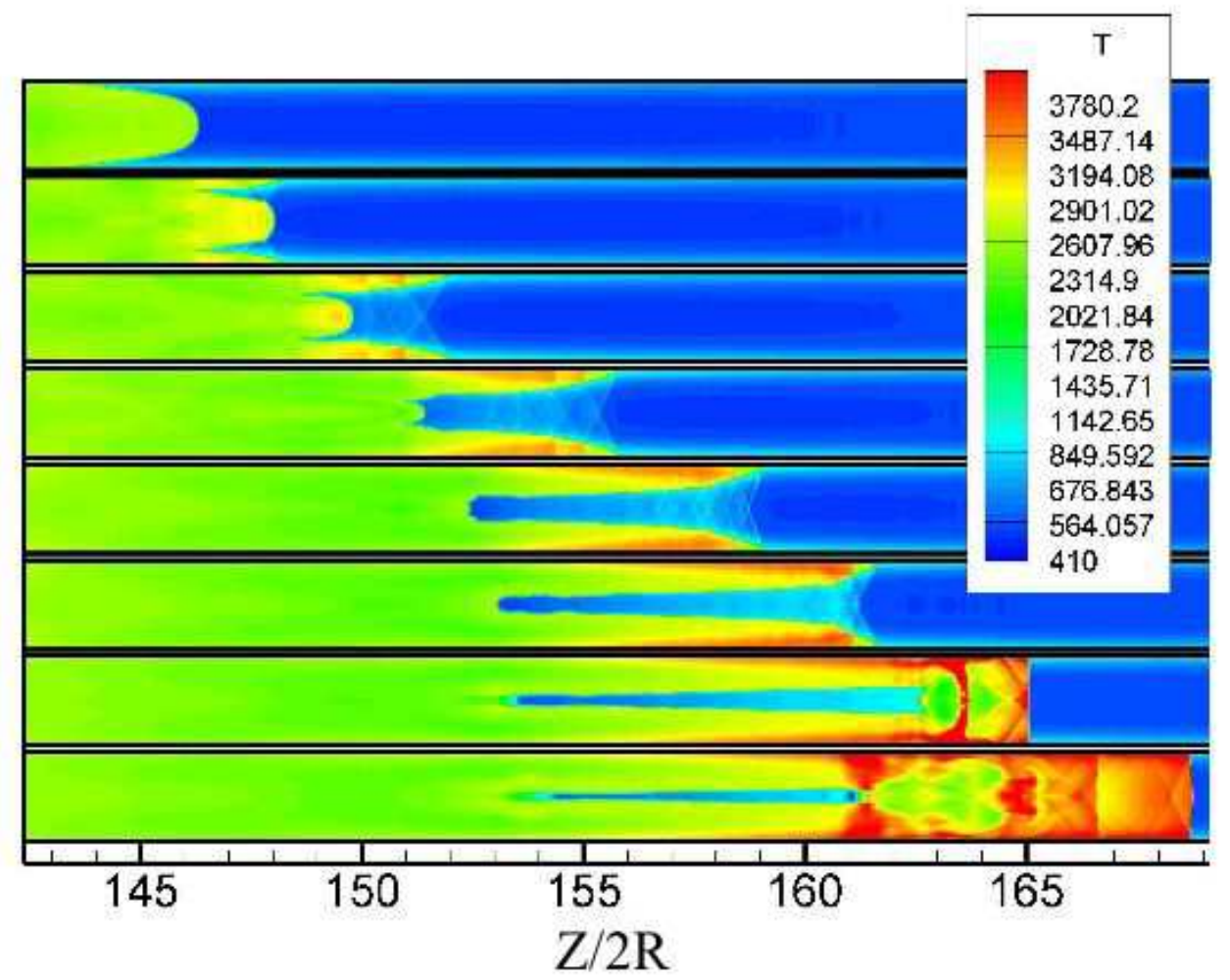

FIG. 9: Temperature field during DDT for the time period from $U_{f} t / R=4.38$ to $U_{f} t / R=4.58$ with equal time intervals between the snapshots. 